Mathematical Modelling and Analysis

Volume 21 Number 1, January 2016, 63-82

http://dx.doi.org/10.3846/13926292.2016.1132787

(c) Vilnius Gediminas Technical University, 2016
Publisher: Taylor\&Francis and VGTU

http://www.tandfonline.com/TMMA

ISSN: $1392-6292$

eISSN: $1648-3510$

\title{
On Strong Convergence of Halpern's Method for Quasi-Nonexpansive Mappings in Hilbert Spaces
}

\section{Jesús Garcia Falset ${ }^{a}$, Enrique Llorens-Fuster ${ }^{a}$, Giuseppe Marino $^{b, c}$ and Angela Rugiano ${ }^{b}$}

\author{
${ }^{a}$ Departámento de Análisis Matemático, Universitát de Valencia \\ Dr. Moliner 50, 46100 Burjassot, Valencia, Spain \\ ${ }^{b}$ Dipartimento di Matematica, Universitá della Calabria \\ 87036 Arcavacata di Rende (CS), Italy \\ ${ }^{c}$ Department of Mathematics, King Abdulaziz University \\ P.O. Box 80203, 21589 Jeddah, Saudi Arabia \\ E-mail(corresp.): jesus.garcia@uv.es \\ E-mail: enrique.llorens@uv.es \\ E-mail: giuseppe.marino@unical.it \\ E-mail: rugiano@mat.unical.it
}

Received June 16, 2015; revised December 10, 2015; published online January 15, 2016

\begin{abstract}
In this paper, we introduce a Halpern's type method to approximate common fixed points of a nonexpansive mapping $T$ and a strongly quasi-nonexpansive mappings $S$, defined in a Hilbert space, such that $I-S$ is demiclosed at 0 . The result shows as the same algorithm converges to different points, depending on the assumptions of the coefficients. Moreover, a numerical example of our iterative scheme is given.
\end{abstract}

Keywords: approximation algorithm, fixed point, variational inequality.

AMS Subject Classification: 47J20; 47J25; 49J10; 65J15.

\section{Introduction}

Let $H$ be a real Hilbert space with the inner product $\langle\cdot, \cdot\rangle$, which induces the norm $\|\cdot\|$. Let $C$ be a nonempty, closed and convex subset of $H$. Let $T$ be a mapping of $C$ into itself; we denote by $F i x(T)$ the set of fixed points of $T$, that is, $\operatorname{Fix}(T)=\{z \in C: T z=z\}$.

We recall that a mapping $T: C \rightarrow H$ is said to be nonexpansive if $\forall x, y \in$ $C$, the following inequality holds

$$
\|T x-T y\| \leq\|x-y\| .
$$


If $T: C \rightarrow H$ is a mapping with $\operatorname{Fix}(T) \neq \emptyset$, then $T$ is said to be quasinonexpansive if, $\forall x \in C, \forall p \in F i x(T)$,

$$
\|T x-p\| \leq\|x-p\| .
$$

Further, the set of fixed points of a quasi-nonexpansive mapping is closed and convex [9].

The problem to approximate fixed points of nonexpansive mappings has been widely investigated by many authors. In the setting of Banach spaces, in 2008, F. Kohsaka and W. Takahashi [10] defined the concept of nonspreading mappings. In the setting of Hilbert spaces, the following characterization of a nonspreading mapping was proved by S. Iemoto and W. Takahashi [8] in 2009. $T: C \rightarrow C$ as a nonspreading mapping if and only if

$$
\|T x-T y\|^{2} \leq\|x-y\|^{2}+2\langle x-T x, y-T y\rangle, \quad \forall x, y \in C .
$$

Observe that if $T$ is a nonspreading mapping from $C$ into itself and $\operatorname{Fix}(T) \neq \emptyset$, then $T$ is quasi-nonexpansive.

Recently, T. Suzuki [16] introduced the concept of Chatterjea mapping.

Let $T$ be a mapping on a subset $C$ of a Banach space $E$ and let $\eta$ be a continuous strictly increasing function from $[0, \infty)$ into itself with $\eta(0)=0$. Then $T$ is called a Chatterjea mapping with respect to $\eta$ if

$$
2 \eta(\|T x-T y\|) \leq \eta(\|T x-y\|)+\eta(\|x-T y\|), \quad \forall x, y \in C .
$$

It is easy to check that a nonspreading mapping on a subset $C$ of a Hilbert space $E$ is a Chatterjea mapping with respect to the function $t \mapsto t^{2}$.

S. Iemoto and W. Takahashi [8] approximated common fixed points of a nonexpansive mapping $T$ and of a nonspreading mapping $S$ in a Hilbert space using Moudafi's iterative scheme [13]. They obtained the following theorem that shows the weak convergence of their iterative method:

Theorem 1. Let $H$ be a Hilbert space and let $C$ be a nonempty closed and convex subset of $H$. Assume that $\operatorname{Fix}(S) \cap F i x(T) \neq \emptyset$. Define a sequence $\left(x_{n}\right)$ as follows:

$$
\left\{\begin{array}{l}
x_{1} \in C, \\
x_{n+1}=\left(1-\alpha_{n}\right) x_{n}+\alpha_{n}\left[\beta_{n} S x_{n}+\left(1-\beta_{n}\right) T x_{n}\right],
\end{array}\right.
$$

where $\left(\alpha_{n}\right),\left(\beta_{n}\right)$ are in $[0,1]$. Then, the following hold:

(i) If $\liminf _{n \rightarrow \infty} \alpha_{n}\left(1-\alpha_{n}\right)>0$ and $\sum_{n=1}^{\infty}\left(1-\beta_{n}\right)<\infty$, then $\left(x_{n}\right)$ converges weakly to $p \in \operatorname{Fix}(S)$;

(ii) If $\sum_{n=1}^{\infty} \alpha_{n}\left(1-\alpha_{n}\right)=\infty$ and $\sum_{n=1}^{\infty} \beta_{n}<\infty$, then $\left(x_{n}\right)$ converges weakly to $p \in F i x(T)$;

(iii) If $\liminf _{n \rightarrow \infty} \alpha_{n}\left(1-\alpha_{n}\right)>0$ and $\liminf _{n \rightarrow \infty} \beta_{n}\left(1-\beta_{n}\right)>0$, then $\left(x_{n}\right)$ converges weakly to $p \in F i x(S) \cap F i x(T)$. 
In order to overcome the weak convergence in [8], in [6] the authors modified the algorithm (1.3) in a Halpern's type method, using the averaged type mappings $T_{\delta}$, i.e. mapping:

$$
T_{\delta}=(1-\delta) I+\delta T, \quad \delta \in(0,1)
$$

The main theorem of $[6]$ is given below.

Theorem 2. Let $H$ be a Hilbert space and let $C$ be a nonempty closed and convex subset of $H$. Let $T: C \rightarrow C$ be a nonexpansive mapping and let $S$ : $C \rightarrow C$ be a nonspreading mapping such that $F i x(S) \cap F i x(T) \neq \emptyset$. Let $T_{\delta}$ and $S_{\delta}$ be the averaged type mappings. Suppose that $\left(\alpha_{n}\right)$ is a real sequence in $(0,1)$ satisfying the conditions:

$$
\text { 1. } \lim _{n \rightarrow \infty} \alpha_{n}=0, \quad 2 . \sum_{n=1}^{\infty} \alpha_{n}=\infty \text {. }
$$

If $\left(\beta_{n}\right)$ is a sequence in $[0,1]$, we define a sequence $\left(x_{n}\right)$ as follows:

$$
\left\{\begin{array}{l}
x_{1} \in C \\
x_{n+1}=\alpha_{n} u+\left(1-\alpha_{n}\right)\left[\beta_{n} T_{\delta} x_{n}+\left(1-\beta_{n}\right) S_{\delta} x_{n}\right]
\end{array}\right.
$$

then, the following hold:

(i) If $\sum_{n=1}^{\infty}\left(1-\beta_{n}\right)<\infty$, then $\left(x_{n}\right)$ strongly converges to $\bar{p}=P_{F i x(T)} u$ which is the unique solution in Fix $(T)$ of the variational inequality $\langle u-\bar{p}, x-\bar{p}\rangle \leq$ 0 , for all $x \in F i x(T)$.

(ii) If $\sum_{n=1}^{\infty} \beta_{n}<\infty$, then $\left(x_{n}\right)$ strongly converges to $\widehat{p}=P_{\text {Fix }(S)} u$ which is the unique solution in Fix $(S)$ of the variational inequality $\langle u-\widehat{p}, x-\widehat{p}\rangle \leq 0$, for all $x \in F i x(S)$.

(iii) If $\liminf _{n \rightarrow \infty} \beta_{n}\left(1-\beta_{n}\right)>0$, then $\left(x_{n}\right)$ strongly converges to $p_{0}=P_{\text {Fix }(T) \cap F i x(S)} u$ which is the unique solution in Fix $(T) \cap$ Fix $(S)$ of the variational inequality $\left\langle u-p_{0}, x-p_{0}\right\rangle \leq 0$, for all $x \in \operatorname{Fix}(T) \cap F i x(S)$.

The aim of this paper will be to improve the Theorem 2 without using averaged type mappings.

\section{Main Result}

In our result we need of the concept of strongly quasi-nonexpansive mappings.

We recall that the concept of strongly nonexpansive mapping was introduced by Bruck and Reich in 1977 [5], as follows: a mapping $T$ is said strongly nonexpansive if $T$ is nonexpansive and whenever $\left(x_{n}-y_{n}\right)$ is bounded and $\left\|x_{n}-y_{n}\right\|-\left\|T x_{n}-T y_{n}\right\| \rightarrow 0$, it follows that $\left(x_{n}-y_{n}\right)-\left(T x_{n}-T y_{n}\right) \rightarrow 0$. 
To our knowledge, Saejung [15] in 2010 introduced the concept of strong quasi-nonexpansivity: a mapping $S$ is said strongly quasi-nonexpansive if $\operatorname{Fix}(S) \neq \emptyset, S$ is quasi-nonexpansive and $x_{n}-S x_{n} \rightarrow 0$ whenever $\left(x_{n}\right)$ is a bounded sequence such that $\left\|x_{n}-p\right\|-\left\|S x_{n}-p\right\| \rightarrow 0$ for some $p \in \operatorname{Fix}(S)$.

In [5] it was proved that an averaged mapping of a nonexpansive mapping defined on a uniformly convex Banach space is strongly nonexpansive.

Remark 1. Following the same line on the proof in [5], one can show that an averaged type mapping $S_{\delta}=(1-\delta) I+\delta S$ of a quasi-nonexpansive mapping is strongly quasi-nonexpansive.

K. Aoyama, S. Iemoto, F. Kohsaka and W. Takahashi [1] first introduced the class of $L$-hybrid mappings in Hilbert spaces. Let $T: H \rightarrow H$ be a mapping and $L \geq 0$ a nonnegative number. $T$ is said $L$-hybrid, signified as $T \in \mathcal{H}_{L}$, if

$$
\|T x-T y\|^{2} \leq\|x-y\|^{2}+L\langle x-T x, y-T y\rangle, \quad \forall x, y \in H .
$$

Notice that for particular choices of $L$ we obtain several important classes of nonlinear mappings. In fact

- $\mathcal{H}_{0}$ is the class of the nonexpansive mappings;

- $\mathcal{H}_{2}$ is the class of the nonspreading mappings;

- $\mathcal{H}_{1}$ is the class of the hybrid mappings.

Further, by the quasi-nonexpansivity of $L$-hybrid mappings, it follows that the assumption on $S$ which is strongly quasi-nonexpansive mapping is weaker than the hypothesis averaged type nonspreading (or also $L$-hybrid). Of course, one can ask if some important $L$-hybrid mappings, as a nonspreading mapping, or a nonexpansive mapping, are already strongly quasi-nonexpansive. This is not always true, as shown in the following example.

Example 1. There exist nonexpansive mappings that are not strongly quasinonexpansive. Moreover, there exist nonspreading mappings that are not strongly quasi-nonexpansive. Let $T: H \rightarrow H$ be such that $T x=-x$. Then $T$ is nonexpansive but not strongly quasi-nonexpansive.

Moreover let $X=A \cup B \cup C \subset H$, where

$$
\begin{aligned}
& A=\{x \in H:\|x\| \leq 1\} ; \quad B=\{x \in H: 1<\|x\|<2\} \\
& C=\{x \in H: 2 \leq\|x\| \leq 3\}
\end{aligned}
$$

Define $S: X \rightarrow X$ by

$$
S x:= \begin{cases}x, & \text { if } x \in A, \\ x /\|x\|, & \text { if } x \in B, \\ 0, & \text { if } x \in C .\end{cases}
$$

One can see that $S$ is a nonspreading mapping, distinguishing three cases $(x \in$ $A, y \in B),(x \in A, y \in C),(x \in B, y \in C)$. 
To see that $S$ is not strongly quasi-nonexpansive take $x_{0}$ with $\left\|x_{0}\right\|=1$. Then $x_{0} \in F i x(S)$. Moreover, define $z_{n}=\left(2+\frac{1}{n}\right) x_{0}$. Then $S z_{n}=0$ and

$$
\left\|z_{n}-x_{0}\right\|-\left\|S z_{n}-x_{0}\right\|=1+\frac{1}{n}-1 \rightarrow 0
$$

but $S z_{n}-z_{n}=\left(2+\frac{1}{n}\right) x_{0} \rightarrow 2 x_{0}$.

Conversely, we have the

Example 2. There exist strongly quasi-nonexpansive mappings that are not $L$-hybrid mappings for any $L$ (and hence that are not ever type average $S_{\delta}$ with $S L$-hybrid). Let $H=\mathbb{R}$. Define

$$
\begin{aligned}
& T(0)=0, \\
& T(n)=-n+\frac{1}{n+1}, \quad T(-n)=n-\frac{1}{n+1}, \quad \forall n \in \mathbb{N}, n>0 .
\end{aligned}
$$

Then define in linear way $T$ on each interval $[n, n+1], n \in \mathbb{Z}$. One can see easily that $\operatorname{Fix}(T)=0$ and $T$ is strongly quasi-nonexpansive. Moreover, from the fact that for large $n \in \mathbb{Z}, T$ is defined almost as $-I$, one can prove that there can not be $L$-hybrid for any $L \geq 0$.

The solid bases on which our proof rest are given by the following lemmas:

Lemma 1 [Xu's Lemma]. [18] Assume $\left(a_{n}\right)_{n \in \mathbb{N}}$ is a sequence of nonnegative numbers such that

$$
a_{n+1} \leq\left(1-s_{n}\right) a_{n}+s_{n} \sigma_{n}+\gamma_{n}, \quad n \geq 0,
$$

where $\left(s_{n}\right)_{n}$ is a sequence in $[0,1]$ and $\left(\sigma_{n}\right)_{n}$ is a sequence in $\mathbb{R}$ such that,

$$
\text { 1. } \sum_{n=1}^{\infty} s_{n}=\infty ; \quad \text { 2. } \lim \sup _{n \rightarrow \infty} \sigma_{n} \leq 0 ; \quad \text { 3. } \gamma_{n} \geq 0, \quad \sum_{n=1}^{\infty} \gamma_{n}<\infty
$$

then $\lim _{n \rightarrow \infty} a_{n}=0$.

Lemma 2 [Maingé's Lemma]. [11] Let $\left(\gamma_{n}\right)_{n}$ be real sequence that has a subsequence $\left(\gamma_{n_{j}}\right)$ which satisfies $\gamma_{n_{j}}<\gamma_{n_{j}+1}$ for all $j$. Then there exists an increasing sequence of integers $(\tau(n))_{n \geq n_{0}}$ sastisfying:

$$
\begin{aligned}
& \text { 1. } \lim _{n} \tau(n)=+\infty ; \quad \text { 2. } \gamma_{\tau(n)} \leq \gamma_{\tau(n)+1}, \quad \text { for all } n \geq n_{0} \\
& \text { 3. } \gamma_{n} \leq \gamma_{\tau(n)+1}, \quad \text { for all } n \geq n_{0} .
\end{aligned}
$$

For the sake of completeness we recall the definition of demiclosedness.

Definition 1. [14] Let $C$ be a nonempty closed convex subset of $H$. Let $T: C \rightarrow C$ be a mapping such that $F i x(T) \neq \emptyset$. The mapping $I-T$ is said demiclosed at 0 if for every sequence $\left(x_{n}\right)_{n \in N}$ weakly convergent to $p \in H$ such that $x_{n}-T x_{n} \rightarrow 0$, it follows that $p \in F i x(T)$. 
To prove the main result, our reasoning is inspired by the ideas contained in $[7,12,17]$.

Theorem 3. Let $H$ be a Hilbert space and let $C$ be a closed convex subset of $H$. Let $T: C \rightarrow C$ be a nonexpansive mapping and $S: C \rightarrow C$ be a strongly quasi-nonexpansive mapping such that $I-S$ is demiclosed in 0 . Assume that $\operatorname{Fix}(T) \cap \operatorname{Fix}(S) \neq \emptyset$. Let $u$ be a fixed anchor in $C$. If $\left(\alpha_{n}\right)_{n},\left(\beta_{n}\right)_{n}$ are sequences in $[0,1]$, we define a sequence $\left(x_{n}\right)_{n \in N}$

$$
\left\{\begin{array}{l}
x_{1} \in C, \\
x_{n+1}=\alpha_{n} u+\left(1-\alpha_{n}\right)\left[\beta_{n} T x_{n}+\left(1-\beta_{n}\right) S x_{n}\right], \quad n \geq 1 .
\end{array}\right.
$$

Then

(1) If $\sum_{n}\left(1-\beta_{n}\right)<\infty, \alpha_{n} \rightarrow 0, \sum_{n} \alpha_{n}=\infty, \sum_{n}\left|\alpha_{n}-\alpha_{n+1}\right|<\infty$, then $\left(x_{n}\right)$ strongly converges to $\bar{p} \in F i x(T)$ that is the unique point in Fix $(T)$ that solves the variational inequality

$$
\langle\bar{p}-u, x-\bar{p}\rangle \geq 0, \quad \forall x \in F i x(T),
$$

i.e. $\bar{p}=P_{F i x(T)} u$.

(2) If $\sum_{n} \beta_{n}<\infty, \alpha_{n} \rightarrow 0, \sum_{n} \alpha_{n}=+\infty, \frac{\beta_{n}}{\alpha_{n}} \rightarrow 0$, then $\left(x_{n}\right)_{n}$ converges strongly to $\tilde{p} \in \operatorname{Fix}(S)$ that is the unique solution in $\operatorname{Fix}(S)$ of the variational inequality

$$
\langle\tilde{p}-u, x-\tilde{p}\rangle \geq 0, \quad \forall x \in F i x(S),
$$

i.e. $\tilde{p}=P_{\text {Fix }(S)} u$.

(3) If $\liminf _{n} \beta_{n}\left(1-\beta_{n}\right)>0, \alpha_{n} \rightarrow 0, \sum_{n} \alpha_{n}=+\infty$, then $\left(x_{n}\right)$ strongly converges to $p_{0} \in F i x(T) \cap F i x(S)$ is the unique solution in Fix $(T) \cap$ $\operatorname{Fix}(S)$ of the variational inequality

$$
\left\langle p_{0}-u, x-p_{0}\right\rangle \geq 0, \quad \forall x \in F i x(T) \cap \operatorname{Fix}(S),
$$

i.e. $p_{0}=P_{F i x(T) \cap F i x(S)} u$.

Proof. In the sequel, we denote by $O(1)$ any bounded real sequence (so, for example, $O(1)+O(1)=O(1))$. First of all, we check that $\left(x_{n}\right)_{n \in N}$ is a bounded sequence. Indeed, let $\left(U_{n}\right)_{n \in N}$ a sequence defined by $U_{n}=\beta_{n} T+\left(1-\beta_{n}\right) S$ and $z \in F i x(T) \cap F i x(S)$. Then,

$$
\begin{aligned}
\left\|x_{n+1}-z\right\| & =\left\|\alpha_{n} u+\left(1-\alpha_{n}\right) U_{n} x_{n}-z\right\| \\
& =\left\|\left(1-\alpha_{n}\right)\left(U_{n} x_{n}-z\right)+\alpha_{n}(u-z)\right\| \\
& \leq\left(1-\alpha_{n}\right)\left\|x_{n}-z\right\|+\alpha_{n}\|u-z\| \\
\text { (by convexity) } & \leq \max \left\{\left\|x_{n}-z\right\|,\|u-z\|\right\} \\
\text { (from induction) } & \leq \max \left\{\left\|x_{1}-z\right\|,\|u-z\|\right\} .
\end{aligned}
$$

Then, $\left(x_{n}\right)$ is bounded. 
Moreover

$$
x_{n+1}-U_{n} x_{n}=\alpha_{n}\left(u-U_{n} x_{n}\right) \rightarrow 0, \quad n \rightarrow \infty,
$$

since $\alpha_{n} \rightarrow 0$.

Proof. (1) The the key will be to prove that $x_{n+1}-x_{n} \rightarrow 0$. In order to show this, we calculate

$$
\begin{aligned}
\left\|x_{n+1}-x_{n}\right\| & =\|\left(1-\alpha_{n}\right)\left(U_{n} x_{n}-U_{n-1} x_{n-1}\right) \\
- & \left(\alpha_{n}-\alpha_{n-1}\right) U_{n-1} x_{n-1}+\left(\alpha_{n}-\alpha_{n-1}\right) u \| \\
& =\left\|\left(1-\alpha_{n}\right)\left(U_{n} x_{n}-U_{n-1} x_{n-1}\right)+\left(\alpha_{n-1}-\alpha_{n}\right)\left(U_{n-1} x_{n-1}-u\right)\right\| \\
& =\|\left(\alpha_{n-1}-\alpha_{n}\right)\left(U_{n-1} x_{n-1}-u\right) \\
& +\left(1-\alpha_{n}\right)\left[\beta_{n} T x_{n}+\left(1-\beta_{n}\right) S x_{n}-\beta_{n-1} T x_{n-1}\right. \\
& \left.-\left(1-\beta_{n-1}\right) S x_{n-1}\right] \| \\
& =\|\left(\alpha_{n-1}-\alpha_{n}\right)\left(U_{n-1} x_{n-1}-u\right) \\
& +\left(1-\alpha_{n}\right)\left[\beta_{n}\left(T x_{n}-T x_{n-1}\right)+\left(1-\beta_{n}\right)\left(S x_{n}-S x_{n-1}\right)\right. \\
& \left.+\left(\beta_{n}-\beta_{n-1}\right) T x_{n-1}+\left(\beta_{n-1}-\beta_{n}\right) S x_{n-1}\right] \| \\
\leq & \left|\alpha_{n-1}-\alpha_{n}\right| O(1) \\
& +\left(1-\alpha_{n}\right)\left[\beta_{n}\left\|x_{n}-x_{n-1}\right\|+\left(1-\beta_{n}\right) O(1)+\left|\beta_{n}-\beta_{n-1}\right| O(1)\right] \\
& =\left(1-s_{n}\right)\left\|x_{n}-x_{n-1}\right\|+\gamma_{n},
\end{aligned}
$$

where $s_{n}=1-\beta_{n}+\alpha_{n} \beta_{n} \geq \alpha_{n} O(1)$ eventually, $\gamma_{n}=\left|\alpha_{n-1}-\alpha_{n}\right| O(1)+[(1-$ $\left.\left.\alpha_{n}\right)\left(1-\beta_{n}\right)+\left|\beta_{n}-\beta_{n-1}\right|\right] O(1)$.

Thanks to hypotheses on $\alpha_{n}, \beta_{n}$ we see that $s_{n} \rightarrow 0, \sum_{n} s_{n}=+\infty$ and $\sum_{n} \gamma_{n}<\infty$. This is sufficient, from Xu's Lemma, to conclude $x_{n+1}-x_{n} \rightarrow 0$. From this and (2.6) follows immediately

$$
x_{n}-U_{n} x_{n} \rightarrow 0,
$$

since $x_{n}-U_{n} x_{n}=\left(x_{n}-x_{n+1}\right)-\left(x_{n+1}-U_{n} x_{n}\right)$. Moreover

$$
\begin{aligned}
\left\|x_{n}-U_{n} x_{n}\right\| & =\left\|x_{n}-\beta_{n} T x_{n}-\left(1-\beta_{n}\right) S x_{n}\right\| \\
& \geq\left\|x_{n}-\beta_{n} T x_{n}\right\|-\left(1-\beta_{n}\right)\left\|S x_{n}\right\|, \\
\left\|x_{n}-\beta_{n} T x_{n}\right\| & \leq\left\|x_{n}-U_{n} x_{n}\right\|+\left(1-\beta_{n}\right) O(1),
\end{aligned}
$$

and so from (2.7) and hypotheses $\sum_{n}\left(1-\beta_{n}\right)<\infty$, we have also $x_{n}-\beta_{n} T x_{n} \rightarrow$ 0 .

From this we deduce also $x_{n}-T x_{n} \rightarrow 0$, and this gives that any weak limit of $\left(x_{n}\right)$ is in Fix $(T)$, since $T$ is nonexpansive, and thus the Principle of Demiclosedness is satisfied.

Now we can show that $x_{n} \rightarrow \bar{p}$, where $\bar{p}$ is the unique solution in Fix $(T)$ of the variational inequality (2.3). We show first that

$$
\limsup _{n}\left\langle U_{n} x_{n}-\bar{p}, u-\bar{p}\right\rangle \leq 0 .
$$


Indeed, let $\left(x_{n_{k}}\right)$ be a subsequence of $\left(x_{n}\right)$ such that

$$
\limsup _{n}\left\langle x_{n}-\bar{p}, u-\bar{p}\right\rangle=\lim _{k}\left\langle x_{n_{k}}-\bar{p}, u-\bar{p}\right\rangle
$$

and $x_{n_{k}} \rightarrow z$. Then $z \in F i x(T)$ and so, from (2.9)

$$
\limsup \left\langle x_{n}-\bar{p}, u-\bar{p}\right\rangle=\langle z-\bar{p}, u-\bar{p}\rangle
$$

and this is nonpositive by definition of $\bar{p}$.

We have

$$
\begin{aligned}
\limsup _{n \rightarrow \infty}\left\langle U_{n} x_{n}-\bar{p}, u-\bar{p}\right\rangle & =\limsup _{n \rightarrow \infty}\left[\left\langle x_{n}-\bar{p}, u-\bar{p}\right\rangle+\left\langle U_{n} x_{n}-x_{n}, u-\bar{p}\right\rangle\right] \\
(\text { from }(2.7)) & =\limsup _{n \rightarrow \infty}\left\langle x_{n}-\bar{p}, u-\bar{p}\right\rangle \\
(\text { by }(2.9)) & \leq 0 .
\end{aligned}
$$

Finally,

$$
\begin{aligned}
\left\|x_{n+1}-\bar{p}\right\| & =\left\|\left(1-\alpha_{n}\right)\left(U_{n} x_{n}-\bar{p}\right)+\alpha_{n}(u-\bar{p})\right\|^{2} \\
& =\left(1-\alpha_{n}\right)^{2}\left\|U_{n} x_{n}-\bar{p}\right\|^{2}+\alpha_{n}^{2}\|u-\bar{p}\|^{2} \\
& +2 \alpha_{n}\left\langle\left(1-\alpha_{n}\right)\left(U_{n} x_{n}-\bar{p}\right), u-\bar{p}\right\rangle \\
& =\left(1-\alpha_{n}\right)^{2}\left\|\beta_{n}\left(T x_{n}-\bar{p}\right)+\left(1-\beta_{n}\right)\left(S x_{n}-\bar{p}\right)\right\|^{2} \\
& +\alpha_{n}^{2}\|u-\bar{p}\|^{2}+2 \alpha_{n}\left\langle U_{n} x_{n}-\bar{p}, u-\bar{p}\right\rangle \\
& -2 \alpha_{n}^{2}\left\langle U_{n} x_{n}-\bar{p}, u-\bar{p}\right\rangle \\
\text { (by } T \text { quasi-nonexp. }) & \leq\left(1-\alpha_{n}\right)^{2}\left[\beta_{n}\left\|x_{n}-\bar{p}\right\|+\left(1-\beta_{n}\right) O(1)\right]^{2}+\alpha_{n}^{2} O(1) \\
& +2 \alpha_{n}\left\langle U_{n} x_{n}-\bar{p}, u-\bar{p}\right\rangle+2 \alpha_{n}^{2} O(1) \\
& \leq\left(1-\alpha_{n}\right)^{2}\left\|x_{n}-\bar{p}\right\|^{2}+\left(1-\beta_{n}\right) O(1)+\alpha_{n}^{2} O(1) \\
& +2 \alpha_{n}\left\langle U_{n} x_{n}-\bar{p}, u-\bar{p}\right\rangle \\
\leq & \left(1-\alpha_{n}\right)^{2}\left\|x_{n}-\bar{p}\right\|^{2}+\left(1-\beta_{n}\right) O(1)+\alpha_{n}^{2} O(1) \\
& +2 \alpha_{n}\left\langle U_{n} x_{n}-\bar{p}, u-\bar{p}\right\rangle \\
& =\left(1-2 \alpha_{n}\right)\left\|x_{n}-\bar{p}\right\|^{2}+\left(1-\beta_{n}\right) O(1)+\alpha_{n}^{2} O(1) \\
& +2 \alpha_{n}\left\langle U_{n} x_{n}-\bar{p}, u-\bar{p}\right\rangle \\
& =\left(1-s_{n}\right)\left\|x_{n}-\bar{p}\right\|^{2}+s_{n} \sigma_{n}+\gamma_{n},
\end{aligned}
$$

where

$$
s_{n}=2 \alpha_{n}, \quad \sigma_{n}=\alpha_{n} O(1)+2\left\langle U_{n} x_{n}-\bar{p}, u-\bar{p}\right\rangle, \quad \gamma_{n}=\left(1-\beta_{n}\right) O(1) .
$$

Thanks to the hypotheses on $\alpha_{n}, \beta_{n}$ and (2.8), from Xu's Lemma again, we obtain $x_{n} \rightarrow \bar{p}$.

Proof. (2) Let $\tilde{p}$ be the unique solution of variational inequality (2.4). We 
want to show that $x_{n} \rightarrow \tilde{p}$. We compute,

$$
\begin{aligned}
\left\|x_{n+1}-\tilde{p}\right\|^{2} & =\left\|\alpha_{n} u+\left(1-\alpha_{n}\right) U_{n} x_{n}-\tilde{p}+\alpha_{n} \tilde{p}-\alpha_{n} \tilde{p}\right\|^{2} \\
& =\left\|\alpha_{n}(u-\tilde{p})+\left(1-\alpha_{n}\right)\left(U_{n} x_{n}-\tilde{p}\right)\right\|^{2} \\
& \leq\left(1-\alpha_{n}\right)^{2}\left\|U_{n} x_{n}-\tilde{p}\right\|^{2}+2 \alpha_{n}\left\langle u-\tilde{p}, x_{n+1}-\tilde{p}\right\rangle \\
& =\left(1-\alpha_{n}\right)^{2}\left\|\beta_{n}\left(T x_{n}-\tilde{p}\right)+\left(1-\beta_{n}\right)\left(S x_{n}-\tilde{p}\right)\right\|^{2} \\
& +2 \alpha_{n}\left\langle u-\tilde{p}, x_{n+1}-\tilde{p}\right\rangle \\
\text { (by } S \text { quasi-nonexp.) } & \leq\left(1-\alpha_{n}\right)^{2}\left\|x_{n}-\tilde{p}\right\|^{2} \\
& +\beta_{n} O(1)+2 \alpha_{n}\left\langle x_{n+1}-\tilde{p}, u-\tilde{p}\right\rangle .
\end{aligned}
$$

At this point we distinguish two cases: or the sequence $\left\|x_{n}-\tilde{p}\right\|$ is eventually not increasing or no.

Alternative 1. $\left(\left\|x_{n}-\tilde{p}\right\|\right)$ is eventually not increasing, so $\left\|x_{n+1}-\tilde{p}\right\| \leq$ $\left\|x_{n}-\tilde{p}\right\|, \forall n \geq N$.

Putting $\sigma_{n}=\left\langle x_{n+1}-\tilde{p}, u-\tilde{p}\right\rangle, \gamma_{n}=\beta_{n} O(1)$ and since $\left(1-\alpha_{n}\right)^{2} \leq\left(1-\alpha_{n}\right)$, we can rewrite $(2.10)$ as $\left\|x_{n+1}-\tilde{p}\right\|^{2} \leq\left(1-\alpha_{n}\right)\left\|x_{n}-\tilde{p}\right\|^{2}+\alpha_{n} \sigma_{n}+\gamma_{n}$, so the thesis $x_{n} \rightarrow \tilde{p}$ will follows again by Xu's Lemma if we are able to show that

$$
\limsup _{n}\left\langle x_{n+1}-\tilde{p}, u-\tilde{p}\right\rangle \leq 0 .
$$

Note that until now we have not used the hypothesis of strong quasi-nonexpansivity of $S$. Now, since $\left(\left\|x_{n}-\tilde{p}\right\|\right)$ is definitively not increasing, there exists the $\lim _{n}\left\|x_{n}-\tilde{p}\right\|$. Then

$$
\begin{aligned}
0 & =\lim _{n \rightarrow \infty}\left(\left\|x_{n+1}-\tilde{p}\right\|-\left\|x_{n}-\tilde{p}\right\|\right) \\
& \leq \liminf _{n \rightarrow \infty}\left(\alpha_{n}\|u-\tilde{p}\|+\left(1-\alpha_{n}\right)\left\|U_{n} x_{n}-\tilde{p}\right\|-\left\|x_{n}-\tilde{p}\right\|\right) \\
\left(\text { by } \alpha_{n} \rightarrow 0\right) & =\liminf _{n \rightarrow \infty}\left(\left\|U_{n} x_{n}-\tilde{p}\right\|-\left\|x_{n}-\tilde{p}\right\|\right) \\
& =\liminf _{n \rightarrow \infty}\left(\left\|\beta_{n}\left(T x_{n}-\tilde{p}\right)+\left(1-\beta_{n}\right)\left(S x_{n}-\tilde{p}\right)\right\|-\left\|x_{n}-\tilde{p}\right\|\right) \\
\left(\text { by } \beta_{n} \rightarrow 0\right) & =\liminf _{n \rightarrow \infty}\left(\left\|S x_{n}-\tilde{p}\right\|-\left\|x_{n}-\tilde{p}\right\|\right) \\
(S \text { quasi-nonexp. }) & \leq \limsup _{n \rightarrow \infty}\left(\left\|x_{n}-\tilde{p}\right\|-\left\|x_{n}-\tilde{p}\right\|\right)=0 .
\end{aligned}
$$

Thus

$$
\lim _{n}\left(\left\|S x_{n}-\tilde{p}\right\|-\left\|x_{n}-\tilde{p}\right\|\right)=0 .
$$

From the strong quasi-nonexpansivity of $S$, we deduce

$$
S x_{n}-x_{n} \rightarrow 0
$$

At this point, by using the demiclosedness of $(I-S)$ in 0 we can proceed as in the Proof of (1) to show (2.11). The statement is proved when the Alternative 1 holds.

Alternative 2. $\left(\left\|x_{n}-\tilde{p}\right\|\right)$ is not definitively not increasing, i.e. there exists a subsequence $\left(\left\|x_{n_{j}}-\tilde{p}\right\|\right)$ such that $\left\|x_{n_{j}}-\tilde{p}\right\|<\left\|x_{n_{j}+1}-\tilde{p}\right\|, \forall j \in \mathbb{N}$. 
From Maingé's Lemma it follows that there exists an increasing sequence of integers $(\tau(n))_{n \in \ltimes}$ satisfying

$$
\begin{aligned}
& \lim _{n} \tau(n)=+\infty, \quad\left\|x_{\tau(n)}-\tilde{p}\right\| \leq\left\|x_{\tau(n)+1}-\tilde{p}\right\|, \\
& \left\|x_{n}-\tilde{p}\right\| \leq\left\|x_{\tau(n)+1}-\tilde{p}\right\|, \quad \forall n \geq n_{0} .
\end{aligned}
$$

Then

$$
0 \leq \liminf _{n}\left(\left\|x_{\tau(n)+1}-\tilde{p}\right\|-\left\|x_{\tau(n)}-\tilde{p}\right\|\right) .
$$

Retracing the same inequalities used to obtain (2.12) with $\tau(n)$ instead of $n$, we obtain

$$
\lim _{n}\left\|S x_{\tau(n)}-\tilde{p}\right\|-\left\|x_{\tau(n)}-\tilde{p}\right\|=0 .
$$

Again the strong quasi-nonexpansivity yields

$$
S x_{\tau(n)}-x_{\tau(n)} \rightarrow 0
$$

and from the demiclosedness of $I-S$ in 0 , we deduce as above

$$
\limsup _{n}\left\langle x_{\tau(n)+1}-\tilde{p}, u-\tilde{p}\right\rangle \leq 0 .
$$

Incidentally, we observe that

$$
\begin{aligned}
\left\|x_{\tau(n)+1}-x_{\tau(n)}\right\| & =\alpha_{\tau(n)}\left\|u-x_{\tau(n)}\right\|+\left(1-\alpha_{\tau(n)}\right) \beta_{\tau(n)} O(1) \\
& +\left(1-\alpha_{\tau(n)}\right)\left\|S x_{\tau(n)}-x_{\tau(n)}\right\|
\end{aligned}
$$

and so, from (2.15) it follows also $x_{\tau(n)+1}-x_{\tau(n)} \rightarrow 0$. We replace in $(2.10) n$ with $\tau(n)$ and we get

$$
\begin{aligned}
\left\|x_{\tau(n)+1}-\tilde{p}\right\| & \leq\left(1-\alpha_{\tau(n)}\right)^{2}\left\|x_{\tau(n)}-\tilde{p}\right\|^{2} \\
& +2 \alpha_{\tau(n)}\left\langle x_{\tau(n)+1}-\tilde{p}, u-\tilde{p}\right\rangle+\beta_{\tau(n)} O(1) \\
\text { (by property }(2.13)) & \leq\left(1-\alpha_{\tau(n)}\right)^{2}\left\|x_{\tau(n)+1}-\tilde{p}\right\|^{2} \\
& +2 \alpha_{\tau(n)}\left\langle x_{\tau(n)+1}-\tilde{p}, u-\tilde{p}\right\rangle+\beta_{\tau(n)} O(1),
\end{aligned}
$$

consequently

$$
\begin{aligned}
2 \alpha_{\tau(n)}\left\|x_{\tau(n)+1}-\tilde{p}\right\|^{2} & \leq\left(\alpha_{\tau(n)}\right)^{2}\left\|x_{\tau(n)+1}-\tilde{p}\right\|^{2} \\
& +2 \alpha_{\tau(n)}\left\langle x_{\tau(n)+1}-\tilde{p}, u-\tilde{p}\right\rangle+\beta_{\tau(n)} O(1)
\end{aligned}
$$

and dividing by $\alpha_{\tau(n)}$, we have

$$
\begin{aligned}
0 & \leq 2\left\|x_{\tau(n)+1}-\tilde{p}\right\|^{2} \leq \alpha_{\tau(n)}\left\|x_{\tau(n)+1}-\tilde{p}\right\|^{2} \\
& +2\left\langle x_{\tau(n)+1}-\tilde{p}, u-\tilde{p}\right\rangle+\frac{\beta_{\tau(n)}}{\alpha_{\tau(n)}} O(1) .
\end{aligned}
$$

Passing to limsup and recalling the hypothesis $\frac{\beta_{n}}{\alpha_{n}} \rightarrow 0$ and (2.16), we obtain $\lim _{n}\left\|x_{\tau(n)}-\tilde{p}\right\| \leq \lim _{n}\left\|x_{\tau(n)+1}-\tilde{p}\right\|=0$. The (2.14) ensures that also $x_{n} \rightarrow \tilde{p}$. 
Proof. (3) Let $p_{0}$ the unique point in $F i x(S) \cap F$ that satisfies the variational inequality (2.5). Then

$$
\begin{aligned}
& \left\|U_{n} x_{n}-p_{0}\right\|^{2}=\left\|\beta_{n}\left(T x_{n}-p_{0}\right)+\left(1-\beta_{n}\right)\left(S x_{n}-p_{0}\right)\right\|^{2} \\
& \quad=\beta_{n}\left\|T x_{n}-p_{0}\right\|^{2}+\left(1-\beta_{n}\right)\left\|S x_{n}-p_{0}\right\|^{2}-\beta_{n}\left(1-\beta_{n}\right)\left\|T x_{n}-S x_{n}\right\|^{2} \\
& \quad \leq\left\|x_{n}-p_{0}\right\|^{2}-\beta_{n}\left(1-\beta_{n}\right)\left\|T x_{n}-S x_{n}\right\|^{2} \\
& \quad\left\|x_{n+1}-p_{0}\right\|^{2}=\left\|\alpha_{n}\left(u-p_{0}\right)+\left(1-\alpha_{n}\right)\left(U_{n} x_{n}-p_{0}\right)\right\|^{2} \\
& \quad \leq\left(1-\alpha_{n}\right)^{2}\left\|U_{n} x_{n}-p_{0}\right\|^{2}+\alpha_{n}^{2} O(1)+\alpha_{n} O(1) \\
& \quad(\text { by }(2.17)) \leq\left\|x_{n}-p_{0}\right\|^{2}-\beta_{n}\left(1-\beta_{n}\right)\left\|T x_{n}-S x_{n}\right\|^{2}+\alpha_{n}^{2} O(1) \\
& \quad+\alpha_{n} O(1) .
\end{aligned}
$$

Also now we distinguish two cases.

Alternative 1. $\left(\left\|x_{n}-p_{0}\right\|\right)$ is eventually not increasing, $\left\|x_{n+1}-p_{0}\right\| \leq$ $\left\|x_{n}-p_{0}\right\|, \forall n \geq N$.

Then there exists $\lim _{n}\left\|x_{n}-\tilde{p}\right\|$, so (2.18) furnish

$$
\beta_{n}\left(1-\beta_{n}\right)\left\|T x_{n}-S x_{n}\right\| \leq\left\|x_{n}-p_{0}\right\|^{2}-\left\|x_{n+1}-p_{0}\right\|^{2}+\alpha_{n}^{2} O(1)+\alpha_{n} O(1)
$$

and so, by hypothesis $\liminf _{n} \beta_{n}\left(1-\beta_{n}\right)>0$, we deduce

$$
T x_{n}-S x_{n} \rightarrow 0 .
$$

Moreover,

$$
\begin{aligned}
0 & =\lim _{n \rightarrow \infty}\left(\left\|x_{n+1}-p_{0}\right\|-\left\|x_{n}-p_{0}\right\|\right) \\
& \leq \liminf _{n \rightarrow \infty}\left(\alpha_{n}\left\|u-p_{0}\right\|+\left(1-\alpha_{n}\right)\left\|U_{n} x_{n}-p_{0}\right\|-\left\|x_{n}-p_{0}\right\|\right) \\
\left(\text { by } \alpha_{n} \rightarrow 0\right) & =\liminf _{n \rightarrow \infty}\left(\left\|U_{n} x_{n}-p_{0}\right\|-\left\|x_{n}-p_{0}\right\|\right) \\
& =\liminf _{n \rightarrow \infty}\left(\left\|\beta_{n}\left(T x_{n}-p_{0}\right)+\left(1-\beta_{n}\right)\left(S x_{n}-p_{0}\right)\right\|-\left\|x_{n}-p_{0}\right\|\right) \\
& \leq \liminf _{n \rightarrow \infty}\left(\beta_{n}\left(\left\|T x_{n}-p_{0}\right\|-\left\|x_{n}-p_{0}\right\|\right)\right. \\
& \left.+\left(1-\beta_{n}\right)\left(\left\|S x_{n}-p_{0}\right\|-\left\|x_{n}-p_{0}\right\|\right)\right) \\
& \leq \limsup _{n \rightarrow \infty}\left(\beta_{n}\left(\left\|T x_{n}-p_{0}\right\|-\left\|x_{n}-p_{0}\right\|\right)\right. \\
& \left.+\left(1-\beta_{n}\right)\left(\left\|S x_{n}-p_{0}\right\|-\left\|x_{n}-p_{0}\right\|\right)\right) \\
(T, S \text { quasi-nonep. }) & \leq 0 .
\end{aligned}
$$

But then

$$
\lim _{n}\left\{\beta_{n}\left[\left\|T x_{n}-p_{0}\right\|-\left\|x_{n}-p_{0}\right\|\right]+\left(1-\beta_{n}\right)\left[\left\|S x_{n}-p_{0}\right\|-\left\|x_{n}-p_{0}\right\|\right]\right\}=0 .
$$

Since both the addends are non positive and the limit of the sum is zero, it follows that

$$
\lim _{n} \beta_{n}\left[\left\|T x_{n}-p_{0}\right\|-\left\|x_{n}-p_{0}\right\|\right]=\lim _{n}\left(1-\beta_{n}\right)\left[\left\|S x_{n}-p_{0}\right\|-\left\|x_{n}-p_{0}\right\|\right]=0
$$


But then the hypothesis $\liminf _{n} \beta_{n}\left(1-\beta_{n}\right)>0$ implies

$$
\lim _{n}\left\|T x_{n}-p_{0}\right\|-\left\|x_{n}-p_{0}\right\|=\lim _{n}\left\|S x_{n}-p_{0}\right\|-\left\|x_{n}-p_{0}\right\|=0
$$

From strong quasi-nonexpansivity of $S$ it follows

$$
S x_{n}-x_{n} \rightarrow 0
$$

Again $x_{n}-T x_{n}=x_{n}-S x_{n}+S x_{n}-T x_{n}$, so by (2.19) and (2.20),

$$
x_{n}-T x_{n} \rightarrow 0 \text {. }
$$

We show now that

$$
\limsup _{n}\left\langle x_{n}-p_{0}, u-p_{0}\right\rangle \leq 0
$$

Indeed, select a subsequence $x_{n_{k}} \rightarrow z$ and such that

$$
\limsup _{n}\left\langle x_{n}-p_{0}, u-p_{0}\right\rangle=\lim _{k}\left\langle x_{n_{k}}-p_{0}, u-p_{0}\right\rangle=\left\langle z-p_{0}, u-p_{0}\right\rangle
$$

But by the demiclosedness of both $T$ and $S$ and by (2.22) and (2.23), one deduces that $z \in F i x(S) \cap F i x(T)$, and so, by definition of $p_{0},(2.24)$ is obtained. Moreover, from (2.22) and (2.23) at once follows,

$$
U_{n} x_{n}-x_{n} \rightarrow 0 \text {. }
$$

Finally we are able to show $x_{n} \rightarrow p_{0}$. Indeed,

$$
\begin{aligned}
& \left\|x_{n+1}-p_{0}\right\|^{2}=\left\|\left(1-\alpha_{n}\right)\left(U_{n} x_{n}-p_{0}\right)+\alpha_{n}\left(u-p_{0}\right)\right\|^{2} \\
& =\left(1-\alpha_{n}\right)^{2}\left\|U_{n} x_{n}-p_{0}\right\|^{2}+\alpha_{n}^{2}\left\|u-p_{0}\right\|^{2}+2 \alpha_{n}\left\langle\left(1-\alpha_{n}\right)\left(U_{n} x_{n}-p_{0}\right), u-p_{0}\right\rangle \\
& \left(U_{n} \text { quasi-nonexp. }\right) \leq\left(1-\alpha_{n}\right)^{2}\left\|x_{n}-p_{0}\right\|^{2}+\alpha_{n}^{2}\left[\left\|u-p_{0}\right\|^{2}-2\left\langle U_{n} x_{n}-p_{0}, u-p_{0}\right\rangle\right] \\
& \quad+2 \alpha_{n}\left\langle U_{n} x_{n}-x_{n}, u-p_{0}\right\rangle+2 \alpha_{n}\left\langle x_{n}-p_{0}, u-p_{0}\right\rangle \\
& \leq\left(1-\alpha_{n}\right)\left\|x_{n}-p_{0}\right\|^{2}+\alpha_{n}^{2} O(1)+2 \alpha_{n}\left\langle U_{n} x_{n}-x_{n}, u-p_{0}\right\rangle \\
& \quad+2 \alpha_{n}\left\langle x_{n}-p_{0}, u-p_{0}\right\rangle .
\end{aligned}
$$

Put

$$
\begin{aligned}
s_{n} & =1-\left(1-\alpha_{n}\right)^{2} \\
\sigma_{n} & =2\left\langle U_{n} x_{n}-x_{n}, u-p_{0}\right\rangle+2\left\langle x_{n}-p_{0}, u-p_{0}\right\rangle+\alpha_{n} O(1),
\end{aligned}
$$

the thesis follows again by Xu's Lemma, taking account of (2.24) and (2.25).

Alternative 2. $\left(\left\|x_{n}-p_{0}\right\|\right)$ is not egventually not increasing, i.e. there exists a subsequence $\left(\left\|x_{n_{j}}-p_{0}\right\|\right)$ such that $\left\|x_{n_{j}}-p_{0}\right\|<\left\|x_{n_{j+1}}-p_{0}\right\|, \forall j \in \mathbb{N}$.

From Maingé's Lemma it follows that there exists an increasing sequence 
of integers $(\tau(n))_{n \in \mathbb{N}}$ satisfying (2.13) and (2.14). Then

$$
\begin{aligned}
0 & \leq \liminf _{n \rightarrow \infty}\left(\left\|x_{\tau(n)+1}-p_{0}\right\|-\left\|x_{\tau(n)}-p_{0}\right\|\right) \\
& \leq \limsup _{n \rightarrow \infty}\left(\left\|x_{\tau(n)+1}-p_{0}\right\|-\left\|x_{\tau(n)}-p_{0}\right\|\right) \\
& \leq \limsup _{n \rightarrow \infty}\left(\left\|x_{n+1}-p_{0}\right\|-\left\|x_{n}-p_{0}\right\|\right) \\
& \leq \limsup _{n \rightarrow \infty}\left(\left\|\left(1-\alpha_{n}\right)\left(U_{n} x_{n}-p_{0}\right)+\alpha_{n}\left(u-p_{0}\right)\right\|-\left\|x_{n}-p_{0}\right\|\right) \\
\text { (by } U_{n} \text { quasi-nonexp.) } & \leq \limsup _{n \rightarrow \infty}\left(\left(1-\alpha_{n}\right)\left\|x_{n}-p_{0}\right\|+\alpha_{n} O(1)-\left\|x_{n}-p_{0}\right\|\right) \\
\left(\text { by } \alpha_{n} \rightarrow 0\right) & =0 .
\end{aligned}
$$

Hence

$$
\lim _{n}\left[\left\|x_{\tau(n)+1}-p_{0}\right\|-\left\|x_{\tau(n)}-p_{0}\right\|\right]=0 .
$$

Now, retracing the same inequalities used to obtain $(2.22)$ with $\tau(n)$ instead of $n$, we have

$$
S x_{\tau(n)}-x_{\tau(n)} \rightarrow 0 .
$$

Moreover, we can rewrite (2.18) as

$$
0 \leq \beta_{\tau(n)}\left(1-\beta_{\tau(n)}\right)\left\|T x_{\tau(n)}-S x_{\tau(n)}\right\|^{2} \leq\left\|x_{\tau(n)}-p_{0}\right\|^{2}-\left\|x_{\tau(n)+1}-p_{0}\right\|^{2}+\alpha_{n} O(1),
$$

and so, from (2.26) and the hypothesis $\liminf _{n} \beta_{n}\left(1-\beta_{n}\right)>0$,

$$
T x_{\tau(n)}-S x_{\tau(n)} \rightarrow 0 .
$$

Again,

$$
x_{\tau(n)}-T x_{\tau(n)}=x_{\tau(n)}-S x_{\tau(n)}+S x_{\tau(n)}-T x_{\tau(n)},
$$

so, by $(2.27)$ and $(2.28), x_{\tau(n)}-T x_{\tau(n)} \rightarrow 0$, so also

$$
x_{\tau(n)}-U_{\tau(n)} x_{\tau(n)} \rightarrow 0 .
$$

The same reasoning used to have $(2.24)$ can be now repeated with $\tau(n)$ instead of $n$ obtaining $\lim \sup _{n}\left\langle x_{\tau(n)}-p_{0}, u-p_{0}\right\rangle \leq 0$. Following the same line to prove (2.24), we replace $n$ with $\tau(n)$ and we obtain

$$
\limsup _{n \rightarrow \infty}\left\langle x_{\tau(n)}-p_{0}, u-p_{0}\right\rangle \leq 0 .
$$

We compute,

$$
\begin{aligned}
\left\|x_{\tau(n)+1}-p_{0}\right\| & \leq\left(1-\alpha_{\tau(n)}\right)^{2}\left\|x_{\tau(n)}-p_{0}\right\|^{2}+\alpha_{\tau(n)}^{2} O(1) \\
& +2 \alpha_{\tau(n)}\left\langle U_{\tau(n)} x_{\tau(n)}-x_{\tau(n)}, u-p_{0}\right\rangle \\
& +2 \alpha_{\tau(n)}\left\langle x_{\tau(n)}-p_{0}, u-p_{0}\right\rangle \\
\text { (by property }(2.13)) & \leq\left(1-\alpha_{\tau(n)}\right)^{2}\left\|x_{\tau(n)+1}-p_{0}\right\|^{2}+\alpha_{\tau(n)}^{2} O(1) \\
& +2 \alpha_{\tau(n)}\left\langle U_{\tau(n)} x_{\tau(n)}-x_{\tau(n)}, u-p_{0}\right\rangle \\
& +2 \alpha_{\tau(n)}\left\langle x_{\tau(n)}-p_{0}, u-p_{0}\right\rangle,
\end{aligned}
$$


consequently

$$
\begin{aligned}
2 \alpha_{\tau(n)}\left\|x_{\tau(n)+1}-p_{0}\right\|^{2} & \leq \alpha_{\tau(n)}^{2} O(1)+2 \alpha_{\tau(n)}\left\langle x_{\tau(n)}-p_{0}, u-p_{0}\right\rangle \\
& +2 \alpha_{\tau(n)}\left\langle U_{\tau(n)} x_{\tau(n)}-x_{\tau(n)}, u-p_{0}\right\rangle
\end{aligned}
$$

dividing by $\alpha_{\tau(n)}$, we get

$$
\begin{aligned}
0 & \leq 2\left\|x_{\tau(n)+1}-p_{0}\right\|^{2} \leq \alpha_{\tau(n)} O(1) \\
& +2\left\langle U_{\tau(n)} x_{\tau(n)}-x_{\tau(n)}, u-p_{0}\right\rangle+2\left\langle x_{\tau(n)}-p_{0}, u-p_{0}\right\rangle .
\end{aligned}
$$

Taking the limsup and recalling the hypothesis (2.29) and (2.30), we obtain

$$
\lim _{n \rightarrow \infty}\left\|x_{\tau(n)}-p_{0}\right\| \leq \lim _{n \rightarrow \infty}\left\|x_{\tau(n)+1}-p_{0}\right\|=0 .
$$

Once again by (2.14) we deduce $x_{n} \rightarrow p_{0}$.

Remark 2. We can show that the same thesis of Theorem 3 holds for averaged type mappings of two quasi-nonexpansive mappings, as a consequence of Remark 1 and using the following inequality established in [17]

$$
\left\langle x-T_{\delta} x, x-p\right\rangle \geq \frac{\delta}{2}\|x-T x\|^{2}, \quad \forall x \in C, p \in \operatorname{Fix}(T)
$$

instead of the (1/2)-inverse strong monotonicity of the mapping $I-T$ when $\mathrm{T}$ is a nonexpansive mapping. Precisely, if we replace in the scheme (2.2) the mappings $T, S$ with the averaged type mappings $T_{\delta}, S_{\delta}$, we obtain the same thesis of Theorem 3 under the assumptions:

- $T, S: C \rightarrow C$ be two quasi-nonexpansive mappings such that $I-T, I-S$ are demiclosed at 0 ;

- $\operatorname{Fix}(S) \cap \operatorname{Fix}(T) \neq \emptyset$;

- same hypotheses on the coefficients $\left(\alpha_{n}\right)_{n \in N}$ and $\left(\beta_{n}\right)_{n \in N}$;

for a sequence $\left(x_{n}\right)$ generated by the Algorithm

$$
\left\{\begin{array}{l}
x_{1} \in C, \\
x_{n+1}=\alpha_{n} u+\left(1-\alpha_{n}\right)\left[\beta_{n} T_{\delta} x_{n}+\left(1-\beta_{n}\right) S_{\delta} x_{n}\right] .
\end{array}\right.
$$

It should be noted that the hypotheses of quasi-nonexpansivity and demiclosedness on the mappings involved in our algorithm (2.32) are independent (see Example 3).

Remark 3. In the literature, there exist some interesting mappings $T$ which are quasi-nonexpansive and such that $I-T$ are demiclosed at 0 . Let $H$ be a real Hilbert space, let $C$ be a nonempty, closed and convex subset of $H$ and $T: C \rightarrow C$ such that $\operatorname{Fix}(T) \neq \emptyset$. Next, we list some examples of such mappings.

1. $\quad T$ nonexpansive mapping, [3]; $\quad 2 . \quad T$ nonspreading mapping, [8];

3. T Chatterjea mapping, [16]; $\quad$ 4. T L-hybrid mapping, [8].

Further, there exist mappings $T$ such that $I-T$ are demiclosed at 0 but not necessarily quasi-nonexpansive: 
(a) [2] $T$ continuous pseudocontractive mapping, i.e. if $\forall x, y \in C$,

$$
\|T x-T y\|^{2} \leq\|x-y\|^{2}+\|(I-T) x-(I-T) y\|^{2} ;
$$

(b) [4] $T k$-strictly pseudononspreading mapping, i.e. if there exists $k \in[0,1)$ such that for all $x, y \in D(T)$

$$
\|T x-T y\|^{2} \leq\|x-y\|^{2}+k\|x-T x-(y-T y)\|^{2}+2\langle x-T x, y-T y\rangle .
$$

Next, we will give an example of a quasi-nonexpansive mapping which does not satisfy the Demiclosedness Principle.

Example 3. Let

$$
B_{\ell^{2}}^{+}=\left\{\left(x_{i}\right)_{i \in N}: \quad \sum_{i=1}^{\infty} x_{i}^{2} \leq 1, \quad x_{i} \geq 0, i=1,2, \ldots\right\}
$$

be the part of the unit ball of $\ell_{2}$ contained in the positive cone.

Let $T: B_{\ell^{2}}^{+} \rightarrow B_{\ell^{2}}^{+}$be defined by

$$
T x=\left\{\begin{array}{l}
0_{\ell^{2}}, \quad 0 \leq\|x\| \leq \frac{1}{2}, \\
\frac{x}{2\|x\|}, \quad \frac{1}{2}<\|x\| \leq 1 .
\end{array}\right.
$$

We have $\operatorname{Fix}(T)=\left\{0_{\ell^{2}}\right\}$. It is obvious that $T$ is a quasi-nonexpansive mapping. It is easy to check that $I-T$ is not demiclosed at $0_{\ell^{2}}$.

\section{An example}

In this section, we illustrate our results with a numerical example.

Example 4. Let $T: B_{\ell^{2}}^{+} \rightarrow B_{\ell^{2}}^{+}$be defined by $T(x)=\left(x_{1}-x_{1}^{3}, x_{2}-x_{2}^{3}, \ldots\right)$. Then, $F i x(T)=\left\{0_{\ell^{2}}\right\}$. We denote by $P_{B^{+}\left[0, \frac{1}{2}\right]}$ the metric projection from $\ell^{2}$ onto

$$
B^{+}\left[0, \frac{1}{2}\right]:=\left\{x=\left(x_{i}\right)_{i \in N} \in B_{\ell^{2}}^{+}: \quad\|x\| \leq \frac{1}{2}\right\} .
$$

Let $S: B_{\ell^{2}}^{+} \rightarrow B_{\ell^{2}}^{+}$defined by $S(x)=P_{B^{+}\left[0, \frac{1}{2}\right]} x$. It is obvious that $\operatorname{Fix}(S)=$ $B^{+}\left[0, \frac{1}{2}\right]$. Notice that $\operatorname{Fix}(T) \cap \operatorname{Fix}(S)=\left\{0_{l^{2}}\right\}$. T is a quasi-nonexpansive mapping, for all $x \in B_{l^{2}}^{+}$,

$$
\left\|T x-0_{\ell^{2}}\right\|^{2}=\sum_{n=1}^{\infty}\left(x_{n}-x_{n}^{3}\right)^{2}=\sum_{n=1}^{\infty} x_{n}^{2}\left(1-x_{n}^{2}\right)^{2} \leq\left\|x-0_{\ell^{2}}\right\|^{2} .
$$

To show that $T$ is a pseudo-contractive mapping we will see that $I-T=A$ is accretive, i.e. for all $x, y \in B_{\ell^{2}}^{+}$

$$
\langle A x-A y, x-y\rangle \geq 0 .
$$

If $x \in B_{\ell^{2}}^{+}$, we denote by $x^{3}=\left(x_{1}^{3}, x_{2}^{3}, \ldots\right) \in B_{\ell^{2}}^{+}$. For all $x, y \in B_{\ell^{2}}^{+}$, we claim that

$$
\left\langle x-y, x^{3}-y^{3}\right\rangle \geq 0 .
$$


Indeed, notice that

$$
\left\langle x-y, x^{3}-y^{3}\right\rangle=\sum_{i=1}^{\infty}\left(x_{i}-y_{i}\right)\left(x_{i}^{3}-y_{i}^{3}\right)
$$

is a series of positive terms, since $\left(x_{i}-y_{i}\right)\left(x_{i}^{3}-y_{i}^{3}\right) \geq 0$ for every $i \in N$. It is easy to check that $T$ is continuous. Therefore, from [2], $I-T$ is demiclosed at $0_{\ell_{2}}$.

On the other hand, $T$ is not a nonexpansive mapping. Indeed, for $x=$ $(1,0, \ldots), y=\left(\frac{3}{4}, 0, \ldots\right) \in B_{l^{2}}^{+}$, one has

$$
\|T x-T y\|=\frac{3}{4}-\left(\frac{3}{4}\right)^{3}=\frac{21}{64}>\|x-y\|=\frac{1}{4} .
$$

It is well known that $S$ is a nonexpansive mapping, and hence $I-S$ is demiclosed at 0 (see [3]).

We recall that $x=\left(x_{1}, x_{2}, \ldots\right) \in B_{\ell^{2}}^{+}$and for $k \in\{2,3\}, x^{k}=\left(x_{1}^{k}, x_{2}^{k}, \ldots\right) \in$ $B_{\ell^{2}}^{+}$. Let $T_{\delta}(x)=x-\delta x^{3}$ and $S_{\delta}(x)=(1-\delta) x+\delta P_{B^{+}\left[0, \frac{1}{2}\right]} x$.

Notice that, if $x \in B^{+}\left[0, \frac{1}{2}\right]$, we get $S_{\delta} x=x$.

In the sequel we will consider two cases:

- in the first one we choose $x_{1}, u$ in $\left\{x=\left(x_{i}\right)_{i \in N} \in B_{\ell^{2}}^{+}:\|x\|>\frac{1}{2}\right\}$;

- in the second case we set $x_{1}, u \in B^{+}\left[0, \frac{1}{2}\right]$.

Case 1.

(i) We set $\left(\alpha_{n}\right)_{n \in N}=\left(\frac{1}{n+1}\right)_{n \in N},\left(\beta_{n}\right)_{n \in N}=\left(1-\frac{1}{(n+1)^{2}}\right)_{n \in N}, x_{1}=e_{1}$, $u=e_{2}$ and $\delta=\frac{1}{3}$.

We consider the sequence $\left(x_{n+1}\right)_{n \in N}$ where $x_{n+1}=\left(x_{1}^{n+1}, x_{2}^{n+1}, \ldots\right)$ and

$$
\begin{aligned}
x_{n+1} & =\frac{1}{n+1} e_{2}+\left(1-\frac{1}{n+1}\right) \\
& \times\left[\left(1-\frac{1}{(n+1)^{2}}\right)\left(x_{n}-\frac{1}{3} x_{n}^{3}\right)+\frac{1}{(n+1)^{2}}\left(\frac{2}{3} x_{n}+\frac{1}{3} P_{B^{+}\left[0, \frac{1}{2}\right]} x_{n}\right)\right] .
\end{aligned}
$$

First, we calculate

$$
\begin{aligned}
& x_{1}=(1,0,0, \ldots) ; \quad x_{2}=\left(3.541666666666666 \cdot 10^{-1}, 5 \cdot 10^{-1}, 0, \ldots\right) ; \\
& x_{3}=\left(2.257270516465794 \cdot 10^{-1}, 6.397040059395266 \cdot 10^{-1}, 0, \ldots\right) ; \\
& \ldots, x_{10^{7}}=\left(6.363036990406555 \cdot 10^{-8}, 6.684363438458816 \cdot 10^{-3}, 0, \ldots\right) ; \ldots
\end{aligned}
$$

Next, we compute the norm of $x_{i}$, for $i=1,2,3, \ldots, 10^{7}$ :

$$
\begin{aligned}
& \left\|x_{1}\right\|=1 ; \quad\left\|x_{2}\right\|=6.127267154105309 \cdot 10^{-1} ; \\
& \left\|x_{3}\right\|=6.783611995538479 \cdot 10^{-1} ; \quad\left\|x_{4}\right\|=6.860980019499106 \cdot 10^{-1} ; \\
& \left\|x_{5}\right\|=6.681028956903493 \cdot 10^{-1} ; \quad\left\|x_{6}\right\|=6.445625797917982 \cdot 10^{-1} ; \\
& \ldots, \quad\left\|x_{10}\right\|=5.664753569100269 \cdot 10^{-1} ; \quad \ldots \\
& \left\|x_{100}\right\|=2.886513537288554 \cdot 10^{-1} ; \ldots,\left\|x_{10^{7}}\right\|=6.684363438761673 \cdot 10^{-3}
\end{aligned}
$$


From Remark 2, we know that this algorithm converges to $\bar{p}=\left\{0_{\ell^{2}}\right\}=$ $=P_{F i x(T)} u$.

(ii) We set $\left(\alpha_{n}\right)_{n \in N}=\left(\frac{1}{n+1}\right)_{n \in N},\left(\beta_{n}\right)_{n \in N}=\left(\frac{1}{8^{n}}\right)_{n \in N}, x_{1}=e_{1}, u=e_{2}$ and $\delta=\frac{1}{3}$.

We consider the sequence $\left(x_{n+1}\right)_{n \in N}$ where $x_{n+1}=\left(x_{1}^{n+1}, x_{2}^{n+1}, \ldots\right)$ and

$$
\begin{aligned}
x_{n+1} & =\frac{1}{n+1} e_{2} \\
& +\left(1-\frac{1}{n+1}\right)\left[\frac{1}{8^{n}}\left(x_{n}-\frac{1}{3} x_{n}^{3}\right)+\left(1-\frac{1}{8^{n}}\right)\left(\frac{2}{3} x_{n}+\frac{1}{3} P_{B^{+}\left[0, \frac{1}{2}\right]} x_{n}\right)\right] .
\end{aligned}
$$

From Remark 2, we know that this algorithm converges to

$$
\widehat{p}=P_{F i x(S)} u=\left(0, \frac{1}{2}, 0, \ldots\right) .
$$

(iii) We set $\left(\alpha_{n}\right)_{n \in N}=\left(\frac{1}{n+1}\right)_{n \in N},\left(\beta_{n}\right)_{n \in N}=\left(\frac{9 n}{10 n+1}\right)_{n \in N}, x_{1}=e_{1}$, $u=e_{2}$ and $\delta=\frac{1}{3}$. We consider the sequence $\left(x_{n+1}\right)_{n \in N}$ where $x_{n+1}=$ $\left(x_{1}^{n+1}, x_{2}^{n+1}, \ldots\right)$ and

$$
\begin{aligned}
x_{n+1} & =\frac{1}{n+1} e_{2}+\left(1-\frac{1}{n+1}\right) \\
& \times\left[\left(\frac{9 n}{10 n+1}\right)\left(x_{n}-\frac{1}{3} x_{n}^{3}\right)+\left(\frac{n+1}{10 n+1}\right)\left(\frac{2}{3} x_{n}+\frac{1}{3} P_{B+\left[0, \frac{1}{2}\right]} x_{n}\right)\right] .
\end{aligned}
$$

From Remark 2, we know that this algorithm converges to $p_{0}=\left\{0_{\ell^{2}}\right\}=$ $P_{\text {Fix }(T) \cap F i x(S)} u$.

Case 2.

If we choose $x_{1}, u \in B^{+}\left[0, \frac{1}{2}\right]$, let $\left(\alpha_{n}\right)_{n \in N}$ be a sequence in $(0,1)$ and let $\left(\beta_{n}\right)_{n \in N}$ be a sequence in $[0,1]$, we derive by induction that

$$
0 \leq\left\|x_{n}\right\| \leq \frac{1}{2}, \quad \forall n \in N
$$

For $n=1$, we have $\left\|x_{1}\right\| \leq \frac{1}{2}$. We assume that(3.2) is true for some $k \in N$ and we prove that (3.2) is obtained for some $k+1 \in N$.

$$
\begin{aligned}
& \left\|x_{k+1}\right\| \\
& \quad=\left\|\alpha_{k} u+\left(1-\alpha_{k}\right)\left[\beta_{k}\left(x_{k}-\frac{1}{3} x_{k}^{3}\right)+\left(1-\beta_{k}\right)\left(\frac{2}{3} x_{k}+\frac{1}{3} \operatorname{Proj}_{B\left[0, \frac{1}{2}\right]} x_{k}\right)\right]\right\| \\
& \quad \leq \alpha_{k}\|u\|+\left(1-\alpha_{k}\right)\left\|\beta_{k} x_{k}\left(1-\frac{1}{3} x_{k}^{2}\right)+\left(1-\beta_{k}\right) x_{k}\right\| \\
& \quad \leq \alpha_{k} \frac{1}{2}+\left(1-\alpha_{k}\right)\left[\beta_{k}\left\|x_{k}\left(1-\frac{1}{3} x_{k}^{2}\right)\right\|+\left(1-\beta_{k}\right)\left\|x_{k}\right\|\right] \\
& \quad \leq \alpha_{k} \frac{1}{2}+\left(1-\alpha_{k}\right)\left[\beta_{k} \frac{1}{2}+\left(1-\beta_{k}\right) \frac{1}{2}\right]=\frac{1}{2} .
\end{aligned}
$$


Moreover, $S_{\delta} x_{n}=x_{n}$, for all $n \in N$. Hence, the algorithm (1.4) becomes,

$$
\begin{aligned}
x_{n+1} & =\alpha_{n} u+\left(1-\alpha_{n}\right)\left[\beta_{n}\left(x_{n}-\frac{1}{3} x_{n}^{3}\right)+\left(1-\beta_{n}\right) x_{n}\right] \\
& =\alpha_{n} u+\left(1-\alpha_{n}\right)\left[x_{n}-\frac{1}{3} \beta_{n} x_{n}^{3}\right], \quad \forall n \in N .
\end{aligned}
$$

We fix $u=\left(0, \frac{1}{4}, 0, \ldots\right)$ and $x_{1}=\left(\frac{1}{2}, 0,0, \ldots\right)$ in the following three cases.

(i) We set $\left(\alpha_{n}\right)_{n \in N}=\left(\frac{1}{n+1}\right)_{n \in N}$ and $\left(\beta_{n}\right)_{n \in N}=\left(1-\frac{1}{(n+1)^{2}}\right)_{n \in N}$. We consider the sequence $\left(x_{n+1}\right)_{n \in N}$ where $x_{n+1}=\left(x_{1}^{n+1}, x_{2}^{n+1}, \ldots\right)$ and

$$
x_{n+1}=\frac{1}{n+1} u+\left(1-\frac{1}{n+1}\right)\left[x_{n}-\frac{1}{3}\left(1-\frac{1}{(n+1)^{2}}\right) x_{n}^{3}\right] \text {. }
$$

From Remark 2, we know that the algorithm (3.3) converges to $\bar{p}=\left\{0_{\ell^{2}}\right\}=$ $P_{F i x(T)} u$.

(ii) We set $\left(\alpha_{n}\right)_{n \in N}=\left(\frac{1}{n+1}\right)_{n \in N}$ and $\left(\beta_{n}\right)_{n \in N}=\left(\frac{1}{8^{n}}\right)_{n \in N}$.

We consider the sequence $\left(x_{n+1}\right)_{n \in N}$ where $x_{n+1}=\left(x_{1}^{n+1}, x_{2}^{n+1}, \ldots\right)$ and

$$
x_{n+1}=\frac{1}{n+1} u+\left(1-\frac{1}{n+1}\right)\left[x_{n}-\frac{1}{3}\left(\frac{1}{8^{n}}\right) x_{n}^{3}\right] .
$$

From Remark 2, we know that the algorithm (3.4) converges to $\widehat{p}=P_{F i x(S)} u=$ $u=\left(0, \frac{1}{4}, 0,0, \ldots\right)$.

(iii) We set $\left(\alpha_{n}\right)_{n \in N}=\left(\frac{1}{n+1}\right)_{n \in N}$ and $\left(\beta_{n}\right)_{n \in N}=\left(\frac{9 n}{10 n+1}\right)_{n \in N}$. We consider the sequence $\left(x_{n+1}\right)_{n \in N}$ where $x_{n+1}=\left(x_{1}^{n+1}, x_{2}^{n+1}, \ldots\right)$ and

$$
x_{n+1}=\frac{1}{n+1} u+\left(1-\frac{1}{n+1}\right)\left[x_{n}-\frac{1}{3}\left(\frac{9 n}{10 n+1}\right) x_{n}^{3}\right] .
$$

From Remark 2, we know that the algorithm (3.5) converges to $p_{0}=\left\{0_{\ell^{2}}\right\}=$ $P_{\text {Fix }(T) \cap F i x(S)} u$.

Remark 4. In the example in Case 1 and Case 2 we show that choosing different coefficients $\beta_{n}$ our algorithm converges to the fixed point of $T$ in $(i)$, to the fixed point of $S$ in $(i i)$ and to common fixed point of two mappings in $(i i i)$.

In the first case, using Matlab Code we obtain that the speed of convergence of our iterative scheme to $\left\{0_{\ell^{2}}\right\}$ in $(i)$ is faster than the one in $($ iii $)$.

Moreover, in $(i i)$ the sequence goes fast to $\widehat{p}=P_{F i x(S)} u=\left(0, \frac{1}{2}, 0, \ldots\right)$. If we compare the Case 1 and Case 2 of the previous example, we observe that in (iii) the sequence $\left(x_{n}\right)$ converges to $\left\{0_{\ell^{2}}\right\}$ in Case 2 faster than in Case 1.

Next, we give the Matlab code for the example 4, $(i)$.

format long e

$n=10000000$;

$x=z \operatorname{eros}(n, 2)$;

$x(1,:)=[1,0]$; 


$$
\begin{aligned}
& E=\text { ones }(n, 1) \text {; } \\
& u=[0,1] \text {; } \\
& \text { for } \quad i=1: n \\
& \begin{aligned}
x(i+1,:) & =\left(\frac{1}{1+i}\right) * u \\
& +\left(1-\frac{1}{i+1}\right) *\left(\left(1-\frac{1}{(i+1)^{2}}\right) *\left(x(i,:)-\frac{1}{3} * x(i,:) .^{3}\right)\right. \\
& \left.+\frac{1}{(1+i)^{2}} *\left(\frac{2}{3} * x(i,:)+\frac{x(i,:)}{6 * E(i)}\right)\right)
\end{aligned} \\
& E(i+1)=\operatorname{norm}(x(i+1,:), 2) ; \\
& \text { end } \\
& \operatorname{disp}(\text { ' ') } \\
& \text { disp('sequences') } \\
& \operatorname{disp}(\mathrm{x}) \\
& \operatorname{disp}(\text { 'norm vector') } \\
& \operatorname{disp}(\mathrm{E})
\end{aligned}
$$

\section{Conclusions}

In this paper, we proposed an algorithm which converges to different fixed points, under suitable assumptions on the coefficients involved in the scheme. Moreover, MATLAB programming language has been used to obtain the computational results presented in the example to illustrate the iterative scheme.

\section{References}

[1] K. Aoyama, Sh. Iemoto, F. Kohsaka and W. Takahashi. Fixed point and ergodic theorems for $\lambda$-hybrid mappings in Hilbert spaces. J. Nonlinear Convex Anal., 11(2):335-343, 2010.

[2] Qing bang Zhang and Cao zong Cheng. Strong convergence theorem for a family of Lipschitz pseudocontractive mappings in a Hilbert space. Mathematical and Computer Modelling, 48(3-4):480-485, 2008. http://dx.doi.org/10.1016/j.mcm.2007.09.014.

[3] F.E. Browder. Semicontractive and semiaccretive nonlinear mappings in Banach spaces. Bull. Amer. Math. Soc., 74:660-665, 1968. http://dx.doi.org/10.1090/S0002-9904-1968-11983-4.

[4] F.E Browder and W.V Petryshyn. Construction of fixed points of nonlinear mappings in Hilbert space. Journal of Mathematical Analysis and Applications, 20(2):197-228, 1967. http://dx.doi.org/10.1016/0022-247X(67)90085-6.

[5] R.E. Bruck and S. Reich. Nonexpansive projections and resolvents of accretive operators in Banach spaces. Houston J. Math., 3:459-470, 1977.

[6] F. Cianciaruso, G. Marino, A. Rugiano and B. Scardamaglia. On strong convergence of Halpern's method using averaged type mappings. J. Appl. Math., 2014. http://dx.doi.org/10.1155/2014/473243. 
[7] N. Hussain, G. Marino and Afrah A. N. Abdou. On Mann's method with viscosity for nonexpansive and nonspreading mappings in Hilbert spaces. Abstract and Applied Analysis, 2014, 2014. http://dx.doi.org/10.1155/2014/152530.

[8] Sh. Iemoto and W. Takahashi. Approximating common fixed points of nonexpansive mappings and nonspreading mappings in a Hilbert space. Nonlinear Analysis: Theory, Methods \& Applications, 71(12):e2082-e2089, 2009. http://dx.doi.org/10.1016/j.na.2009.03.064.

[9] Sh. Itoh and W. Takahashi. The common fixed point theory of singlevalued mappings and multivalued mappings. Pacific J. Math., 79(2):493-508, 1978. http://dx.doi.org/10.2140/pjm.1978.79.493.

[10] F. Kohsaka and W. Takahashi. Fixed point theorems for a class of nonlinear mappings related to maximal monotone operators in Banach spaces. Archiv der Mathematik, 91(2):166-177, 2008. http://dx.doi.org/10.1007/s00013-008-25458.

[11] Paul-Emile Maingé. Strong convergence of projected subgradient methods for nonsmooth and nonstrictly convex minimization. Set-Valued Analysis, 16(78):899-912, 2008. http://dx.doi.org/10.1007/s11228-008-0102-z.

[12] G. Marino and Hong-Kun Xu. A general iterative method for nonexpansive mappings in Hilbert spaces. Journal of Mathematical Analysis and Applications, 318(1):43-52, 2006. http://dx.doi.org/10.1016/j.jmaa.2005.05.028.

[13] A. Moudafi. Krasnoselski-Mann iteration for hierarchical fixed-point problems. Inverse Problems, 23(4):1635-1640, 2007. http://dx.doi.org/10.1088/0266$5611 / 23 / 4 / 015$.

[14] Z. Opial. Weak convergence of the sequence of successive approximations for nonexpansive mappings. Bulletin of the American Mathematical Society, 73:591597, 1967. http://dx.doi.org/10.1090/S0002-9904-1967-11761-0.

[15] S. Saejung. Halpern's iteration in Banach spaces. Nonlinear Analysis: Theory, Methods \& Applications, 73(10):3431-3439, 2010. http://dx.doi.org/10.1016/j.na.2010.07.031.

[16] T. Suzuki. Fixed point theorems for a new nonlinear mapping similar to a nonspreading mapping. Fixed Point Theory and Applications, 2014, 2014. http://dx.doi.org/10.1186/1687-1812-2014-47.

[17] K. Wongchan and Satit Saejung. On the strong convergence of viscosity approximation process for quasinonexpansive mappings in Hilbert spaces. Abstract and Applied Analysis, 2011, 2011. http://dx.doi.org/10.1155/2011/385843.

[18] Hong-Kun Xu. Iterative algorithms for nonlinear operators. Journal of the London Mathematical Society, 66(1):240-256, 2002. http://dx.doi.org/10.1112/S0024610702003332. 